\title{
Mindfulness-Based Stress Reduction for the Treatment of Adolescent Psychiatric Outpatients: A Randomized Clinical Trial
}

\author{
Gina M. Biegel \\ Kaiser Permanente Hospital
}

Shauna L. Shapiro
Santa Clara University

\author{
Kirk Warren Brown \\ Virginia Commonwealth University \\ Christine M. Schubert \\ Virginia Commonwealth University
}

\begin{abstract}
Research has shown that mindfulness-based treatment interventions may be effective for a range of mental and physical health disorders in adult populations, but little is known about the effectiveness of such interventions for treating adolescent conditions. The present randomized clinical trial was designed to assess the effect of the mindfulness-based stress reduction (MBSR) program for adolescents age 14 to 18 years with heterogeneous diagnoses in an outpatient psychiatric facility (intent-to-treat $N=102$ ). Relative to treatment-as-usual control participants, those receiving MBSR self-reported reduced symptoms of anxiety, depression, and somatic distress, and increased self-esteem and sleep quality. Of clinical significance, the MBSR group showed a higher percentage of diagnostic improvement over the 5-month study period and significant increases in global assessment of functioning scores relative to controls, as rated by condition-naïve clinicians. These results were found in both completer and intent-to-treat samples. The findings provide evidence that MBSR may be a beneficial adjunct to outpatient mental health treatment for adolescents.
\end{abstract}

Keywords: mindfulness-based stress reduction, mindfulness, adolescents

Over the past 50 years, the prevalence of adolescent psychiatric disorders has risen substantially (e.g., Collishaw, Maughan, Goodman, \& Pickles, 2004). In the United States, it is estimated that 6 to 9 million children and adolescents have a diagnosable mental disorder with at least minimum impairment (U.S. Public Health Service, 2000); 1 in 5 children and adolescents 9 to 17 years old have a mental health disorder that leads to impairment in daily functioning (U.S. Department of Health and Human Services, 1999). Anxiety and mood disorders, particularly depression, are the most frequently reported of these disorders, with combined prevalence rates ranging from $9 \%$ to $15 \%$ in adolescence (e.g., Hyman, 2001; Shaffer et. al., 1996).

Psychotherapeutic treatment of adolescents with mental health problems can be difficult, however (Greene \& Walker, 1997; Hammen, Rudolph, Weisz, Rao, \& Burge, 1999). For example, some adolescents do not view psychotherapy as a beneficial treat-

Gina M. Biegel, Department of Child and Adolescent Psychiatry, Kaiser Permanente Hospital; Kirk Warren Brown, Department of Psychology, Virginia Commonwealth University; Shauna L. Shapiro, Department of Counseling Psychology, Santa Clara University; and Christine M. Schubert, Department of Biostatistics, Virginia Commonwealth University.

We thank Albert Farrell for statistical assistance, Khristina Sanders and Pansy Taylor for research assistance, and Michael Southam-Gerow for helpful comments on a draft of this article.

Correspondence concerning this article should be addressed to Gina M. Biegel, Kaiser Permanente Hospital, 175 Bernal Road, Suite 140, San Jose, CA 95136; or Kirk Warren Brown, Department of Psychology, Virginia Commonwealth University, 806 West Franklin Street, Richmond, VA 23284. E-mail: gina@stressedteens.com or kwbrown@vcu.edu ment option (Walker et al., 1993). In addition, level of response to treatment has also been less than desirable. In a recent review of one commonly used approach for mood disorders, cognitive behavior therapy, Emslie, Mayes, Laptook, and Batt (2003) found that $35 \%-40 \%$ of treated adolescents showed diagnostic remission, leaving 55\%-60\% with continued impairment. This suggests room for new interventions to augment psychological treatment for adolescents (Emslie et al., 2003). Specifically, research suggests a need to implement programs to enhance health promotion, emotional well-being, and use of adaptive coping skills to reduce the use of nonproductive coping skills (e.g., self-harming behaviors; Frydenberg et al., 2004).

The current study was designed to examine the potential efficacy of an intervention that has received considerable attention for the enhancement of cognitive, emotional, and behavioral wellbeing in a variety of adult psychiatric populations-namely, mindfulness-based stress reduction (MBSR). MBSR is a psychoeducational training program in mindfulness and its applications to daily life. Fundamentally, mindfulness involves a sustained, receptive attention to events and experiences on a moment-to-moment basis (e.g., Brown \& Ryan, 2003). Specifically, mindfulness involves giving close attention to stimulus inputs that enter awareness without engaging in discursive thought about them. In MBSR and related contemporary mindfulness-based interventions, training in mindfulness focuses on the development of mindfulness in day-to-day life, while also encouraging an attitude of acceptance or nonjudgmentality toward events and experiences to facilitate the practice of mindful presence (Shapiro, Carlson, Astin, \& Freedman, 2006). 
The vast majority of research testing the efficacy of MBSR and other mindfulness interventions has focused on adult populations. Research has found that such interventions can significantly reduce anxiety and panic symptoms (Miller, Fletcher, \& Kabat-Zinn, 1995), depressive relapse (e.g., Segal, Williams, \& Teasdale, 2002), and psychological distress in both clinical and healthy, stressed populations (e.g., Shapiro, Brown, \& Biegel, 2007). Recent meta-analytic reviews have concluded that on average, mindfulness-based interventions have a medium-sized effect $(d=$ .50 , approximately) on the variety of mental health outcomes that have been examined, including depression, anxiety, sleep quality, and general psychological functioning (Baer, 2003; Grossman, Niemann, Schmidt, \& Walach, 2004).

\section{Mindfulness-Based Interventions with Adolescents and Children}

There has been very little research testing the efficacy of mindfulness-based interventions with adolescents; however, preliminary evidence is suggestive. Bootzin and Stevens (2005) examined the effects of an integrative mindfulness-based intervention with adolescent substance abusers ages 13-19 years. A sixsession intervention that included components of MBSR and insomnia treatment significantly improved sleep and reduced worry and mental health distress. Preliminary research has also explored the efficacy of MBSR with children. Wall (2005) combined MBSR with the practice of Tai Chi with 11 nonclinical, parapubertal children (ages 11-13 years) and found that participants reported increases in well-being, including calmness and relaxation, as well as greater self-care (e.g., improved sleep and concentration), greater self-awareness, and less emotional and behavioral reactivity. In another recent pilot study (Semple, Reid, \& Miller, 2005), 5 children ages 7 to 8 years with anxiety-related academic difficulties received a 6-week mindfulness training program. Participants showed improvements in academic performance and teacherreported problem behavior. Finally, Napoli, Krech, and Holley (2005) used an integrative program of mindfulness and relaxation with 194 children in first to third grade with high anxiety. Participants showed a significant increase in selective attention and decreases in both test anxiety and ADHD behaviors.

These studies are preliminary and have been limited by a number of methodological problems, including small sample sizes, purely self-report outcomes, and a lack of randomized controls. Variations in intervention content have also made it difficult to isolate the effects of mindfulness training. However, this initial research suggests that a mindfulness-based intervention may have value for the treatment of psychological symptoms in adolescents. There are other reasons to suggest that an intervention such as MBSR may be effective in a symptomatic adolescent population. First, as already noted, the MBSR program appears to be adaptable to a range of psychological (and physical) conditions (e.g., Baer, 2003). Second, as training in "present-centered" attention, MBSR may foster more adaptive processing of thoughts and emotions that underlie stress and the psychological and behavioral problems associated with it in adolescents and adults. Specifically, much stress is generated by particular cognitive and emotional responses that involve "time travel" into the remembered past (e.g., rumination) and the imagined future (e.g., anxiety). Through its focus on clear, dispassionate attention to present experience, mindfulness training is thought to foster acceptance, metacognitive awareness, and other processes that help to disengage from cognitive and emotional events that fuel stress and consequent psychological problems (e.g., Baer, 2003; Brown, Ryan, \& Creswell, 2007). Finally, the MBSR emphasis on experiential practice in the deployment of attention in meditative contexts appears to be welltolerated by adolescents (e.g., Barnes, Davis, Murzynowski, \& Treiber, 2004).

\section{Purpose of Study}

The present study sought to extend the incipient research on the use of mindfulness training with adolescents and specifically among a psychologically symptomatic population. A randomized clinical trial tested the efficacy of a modified MBSR program (versus a wait-list control group receiving treatment as usual [TAU]) as an adjunctive treatment for adolescents in an outpatient psychiatry facility. Given the novelty of the MBSR research with adolescent outpatients, we assessed the effect of the intervention on a broad class of indicators relevant to mental health. On the basis of considerable research with adults, we hypothesized that relative to the control condition, the MBSR intervention would positively impact stress-related psychological symptoms, such as depression, anxiety, and sleep difficulties, as well as low selfesteem, which in adolescents has been associated with a host of concurrent and later mental health problems (Boden, Fergusson, \& Horwood, 2008). To objectively assess the efficacy of MBSR, we also tested the effect of the intervention on psychiatric diagnostic outcomes. We hypothesized that MBSR participation would facilitate positive diagnostic change and improve global psychological functioning relative to treatment as usual.

\section{Method}

\section{Participants}

Study participants were recruited from an outpatient child and adolescent psychiatry department through a Kaiser Permanente hospital, part of a national health management organization. Adolescents ages 14-18 years who were currently under psychiatric care or had been so in the past (without time restrictions) were eligible to participate in this study. Exclusion criteria included current substance abuse (either drug or alcohol) or past or present psychiatric or neurological disorders that would preclude or severely limit participation in the study (e.g., currently suicidal, organic brain syndrome, or significant cognitive impairment). Participants were recruited through their primary mental health provider and via flyers posted in the outpatient clinic lobby. All 104 individuals consecutively screened for participation were eligible to participate, and all elected to do so. Sample size was estimated from the results of a power analysis performed using effect size estimates from recent meta-analytic reviews of MBSR (e.g., Grossman et al., 2004). Two participants were noncompliant with completion of the baseline measures, leaving a final intent-to-treat sample of $N=102$. Seventy-four participants completed assessments at all three time points and formed the completer sample.

Figure 1 displays the patient flow through the study. Of the 28 noncompleting participants, 22 were reached after study completion and interviewed by phone regarding their reasons for not 


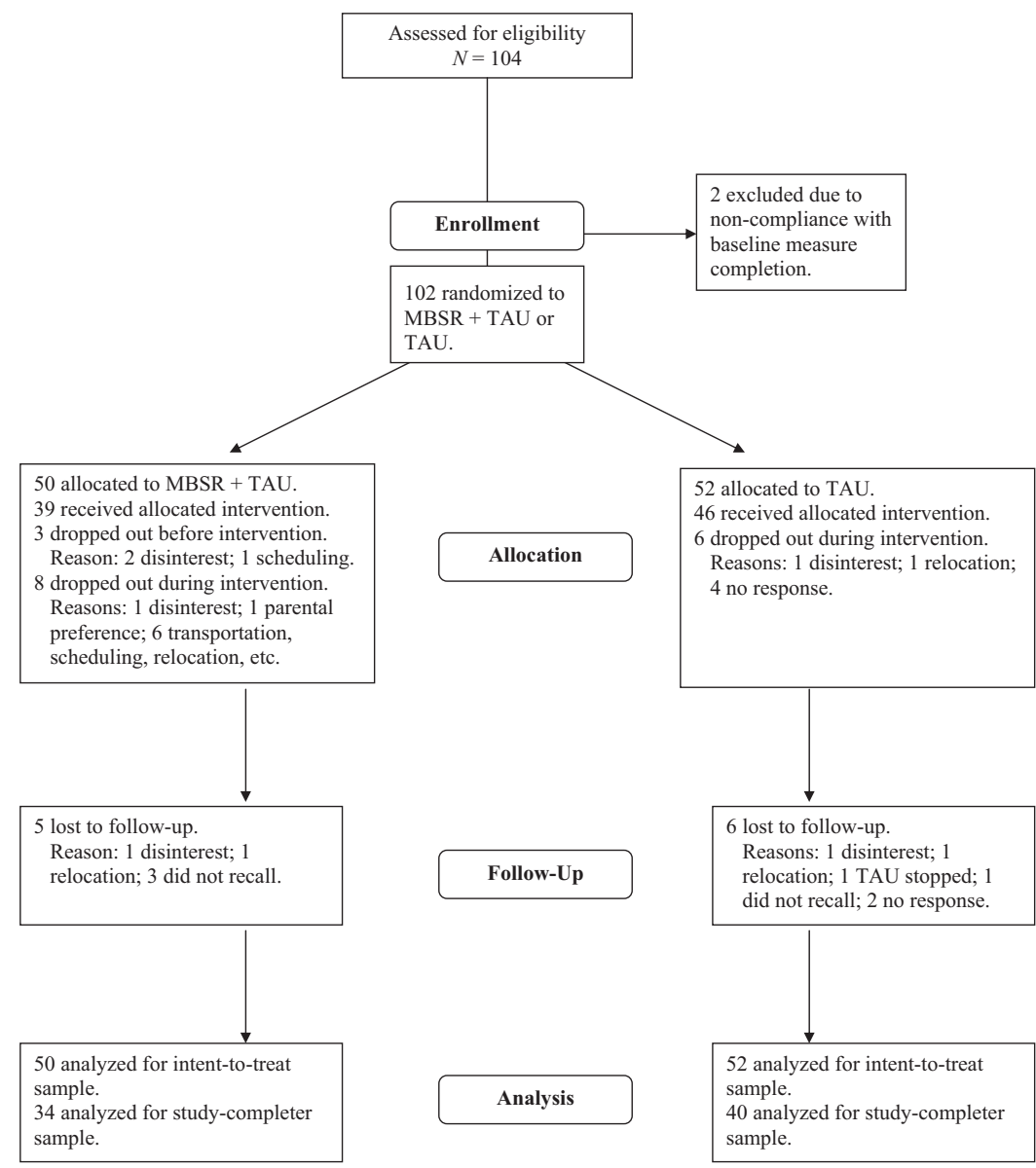

Figure 1. Participant flow through the study. MBSR $=$ mindfulness-based stress reduction; TAU $=$ treatment as usual.

completing the study, using an adaptation of the Reasons for Ending Treatment Questionnaire (RETQ; Garcia \& Weisz, 2002), which is oriented to youth mental health treatment. Among noncompleters allocated to MBSR $(n=16), 3$ indicated disinterest in the program ( 2 of these before beginning), 1 indicated that a parent did not want him/her to continue class attendance, and 1 indicated flagging interest in questionnaire completion at follow-up. Other reasons for noncompletion included transportation difficulties $(n=3)$, scheduling conflicts $(n=3)$, and geographic relocation $(n=2)$. Of the 12 TAU noncompleters, 6 were reached for interview. Two indicated reduced interest in questionnaire packet completion. Other reasons included geographic relocation $(n=2)$, and discontinuation of TAU $(n=1)$. In sum, reasons for noncompletion were varied, and few reflected issues with treatment acceptability or study burden. The study received approval from the hospital's institutional review board, and all participants and one parent provided informed written consent. Random assignment was conducted by Gina M. Biegel using blind selection of condition numbers from a preshuffled bin, with no foreknowledge of participant identifiers or other data. Assignment was concealed from participants until assignment to conditions and completion of the baseline (pretest) measures.

\section{Study Design and Procedures}

The study used a 2 (experimental vs. wait-list control group) by 3 (pretest, posttest, and 3-month follow-up) mixed factorial design. The MBSR intervention was offered as an adjunct to psychiatric treatment. Thus, all participants continued their usual psychological care. Posttest measures were obtained from all available participants 8 weeks later (immediately following MBSR program completion) and at 3 months following the posttest. Thus, the study duration was 5 months ( 20 weeks). Two waves of participant recruitment and data collection were done over a 13-month period, from May 2005 to June 2006.

\section{Intervention: $M B S R$}

This manualized intervention consisted of eight weekly classes, meeting 2 hr per week (e.g., Kabat-Zinn, 1990). Two master'sdegree-level instructors trained in MBSR facilitated both the intervention and wait-list control groups. The primary focus of the classes was on formal and informal mindfulness practices. In these practices, participants were encouraged to bring three core elements to bear: intention, attention, and attitude (Shapiro et al., 2006). Intention refers to the direction of self-endorsed effort 
toward mindfulness practice, which centers on the deployment of mindful attention - the observation of experience as it is occurring in the present moment in a nonevaluative and nondiscursive way. Unlike the typical use of attention, which is tightly intertwined with cognition, mindful attention does not compare, categorize, or ruminate upon events or experiences based on memory. Instead, mindfulness practice is a simple experiencing of what is taking place moment to moment. Finally, the attitudinal element involves a nonjudgmental or open quality brought to moment-to-moment attention and is thought to facilitate sustained attention to what otherwise may be difficult cognitive, emotional, or somatic experiences.

Participants received weekly in-class training in the following formal mindfulness practices: body scan meditation, sitting meditation, Hatha yoga, and walking meditation. These formal practices were designed to enhance an ongoing awareness of momentto-moment mind-body experience, including those of a cognitive, emotional, kinesthetic, and sensory nature. Their purpose was to cultivate the capacity to be mindful during practice sessions and in daily life. Informal practice concerned mindful attention to selected routine, day-to-day activities. The weekly sessions also included didactic presentations, group sharing of practice-related and other experiences, and instruction in at-home mindfulness practice assignments.

Adaptations from the adult MBSR program. In an attempt to develop a course specific to the needs of adolescents, several modifications to the standard MBSR intervention were made (Biegel, 2005). First, at-home mindfulness practices were reduced from 45 min to 20-35 min in length. Further, there was no day-long retreat. Finally, presentations and discussion topics on stress and behavior focused on issues predominant among adolescents, and adolescents with psychiatric disorders more specifically, including self-image, life transitions, self-harming behaviors, and difficulties related to communication and interpersonal relationships.

Training materials. All MBSR participants received a workbook as an adjunct to the group sessions to reinforce the instruction and topics discussed during each of the eight classes. A CD with the sitting and body scan meditations was also provided to facilitate at-home practice.

\section{Treatment as Usual}

TAU involved individual or group psychotherapy and/or psychotropic medication management at the study site. In accord with usual clinical care at the site, there was considerable variance in TAU received.

\section{Study Measures}

Standard demographic measures (gender, age, and ethnicity or race) were obtained at pretest. Except where indicated, the remaining measures were obtained at each of the three assessment points (pretest, posttest, and 3-month follow-up).

Clinical measures of mental health. Information on Diagnostic and Statistical Manual of Mental Disorders (4th ed.; DSM-IVTR; American Psychiatric Association, 2000) psychiatric diagnoses (Axis I: Clinical Disorders) and current level of general psychological and social functioning (Axis V: Global Assessment of Functioning [GAF]) was obtained from institution records for all three time points. These measures of mental health were made by clinicians naïve to participants' treatment condition (blinding was verified by clinician report after study completion). In accord with standard clinical practice and training at the treatment site, diagnostic and GAF data were based on the individual clinicians' reports. At posttest and follow-up, a score was assigned to each participant that reflected change, or lack thereof, in Axis I diagnoses-namely, no change in the number of conditions that each participant was diagnosed with at pretest, an increase in the number of diagnoses (worsened psychiatric condition), or a decrease in the number of diagnoses (improved condition). For example, a participant with both depressive disorder and anxiety disorder at pretest who was no longer diagnosed with an anxiety disorder at posttest would be considered to have an improved diagnostic condition. In actuality, there were few cases of worsened conditions; thus, analyses focused on improvement versus lack of improvement in diagnostic condition. This diagnostic change variable was designed to be conservative, accounting only for the number of diagnoses at each time and not change in the severity of each diagnostic condition.

In addition to $D S M-I V-T R$ Axis $\mathrm{I}$ and $\mathrm{V}$ information, the following information was obtained for three time periods (prestudy, pretest to posttest, and posttest to follow-up): number of individual and group psychotherapy visits, number of mental health hospitalizations, type of mental health medication(s), and medication dosage(s).

Self-report measures of mental health. In an attempt to broadly assess psychological stress, distress, and well being, wellvalidated scales tapping several cognitive and affective dimensions of experience were used. The 10-item version of the Perceived Stress Scale (PSS-10; Cohen \& Williamson, 1988) was used to measure the degree to which situations in one's life during the past month are appraised as stressful on a 5-point scale (never to very often). The internal consistency of the scale (using Cronbach's alpha) in the present sample was .74. Anxiety was measured at both state ("past week") and trait ("past month") levels, using the State/Trait Anxiety Inventory (STAI; Spielberger, 1983). The state STAI (sample $\alpha=.92$ ) and the trait STAI (sample $\alpha=.94$ ) each contained 20 self-descriptive statements rated on a 4-point scale (not at all to very much so).

The Hopkins Symptom Checklist 90 (Revised) nonpatient adolescent measure (SCL-90-R; Derogatis, 1977) assessed psychological symptom patterns on a 5-point scale of distress (not at all to extremely). Six of the nine symptoms subscales were used that were deemed most appropriate to the population of adolescents under study here: Depression, Anxiety, Obsession-Compulsion, Somatization, Interpersonal Sensitivity, and Hostility (sample $\alpha$ range $=.85$ to .90$)$.

The 10-item Rosenberg Self-Esteem Scale (SES; Rosenberg, 1989) was rated on a 4-point Likert scale (strongly agree to strongly disagree). Higher scores reflected lower self-esteem (sample $\alpha=.92$ ). Sleep quality change was assessed with a single item at both posttest and follow-up: "Using the 1 to 7 scale below, please indicate whether there have been any changes in the quality of your sleep since you started the course" (ranging from got much worse to got much better).

Alcohol and illicit drug use was assessed by asking respondents to name the substance (up to three), then indicate whether usage had increased, decreased, or been unchanged since the last assess- 
ment (participants were reminded of the confidential nature of their responses).

Finally, an MBSR program evaluation was completed at posttest that assessed participants' perceptions of program value (yes/no), personal importance (not at all to very important on a 7-point scale) and, in open-ended fashion, perceived benefits to participation and suggestions for program improvement.

\section{Mindfulness Practice Diaries}

MBSR participants completed weekly mindfulness practice diaries during the 8-week intervention and at the 3-month follow-up point to examine the effects of practice on study outcomes. Participants responded to a brief series of questions on each of the mindfulness practices (sitting meditation, body scan meditation, Hatha yoga, and informal mindfulness practice), including the number of days of practice and time spent in practice each week.

\section{Statistical Analyses}

To assess the effect of the MBSR + TAU versus TAU only on self-reported and clinician-rated mental health outcomes, four primary types of analyses were performed. First, for continuous variable outcomes, which included the self-report measures and GAF scores, a restricted maximum likelihood (REML) mixed modeling approach was used (e.g., Bryk \& Raudenbush, 1992; Kreft \& deLeeuw, 1998). In these models, primary interest was in the 2 (treatment condition) $\times 3$ (time) interaction while permitting control of relevant categorical and continuous demographic and clinical variables. The mixed model approach is well suited to hierarchically nested data structures in which a lower level unit of analysis (Level 1; e.g., repeated measures of psychological characteristics) is nested within a higher level of analysis (Level 2; e.g., treatment condition). Other advantages include the ability to retain cases for which missingness is present, which in the present case permitted analysis of the full, intent-to-treat (ITT) sample - that is, using all available data from all randomized subjects as allocated $(N=102)$. The MIXED procedure in SAS (Version 9.1) was used to estimate all REML mixed models.

To enhance interpretability of the mixed model intercept parameters, the predictor variables were pretreated (Bryk \& Raudenbush, 1992; Schwartz \& Stone, 1998): Level 2 continuous variables measured at the beginning of the study, including age and number of prestudy therapy sessions, were centered around zero, whereas Level 2 categorical variables that did not include a meaningful zero value in the original scaling (gender, group, time) were rescaled to include zero. Level 2 variables were treated as fixed effects while the Level 1 time variable was treated as random (as was the intercept and slope for each participant). The between/ within method for computing denominator degrees of freedom was used in all models. Choice of most appropriate within-person error covariance structure (unstructured, compound symmetry, or firstorder autoregressive) was determined through chi-square tests comparing the -2 restricted log likelihood model fit indices for each outcome (as well as significance of autoregression). Unstructured or compound symmetry covariance structures were used in all models. Preliminary REML unconditional means models showed significant between-person variation (individual differences in average values of each outcome) and within-person vari- ation (variations across time within persons) on all outcomes $(p s<.0001)$, supporting the investigation of treatment condition effects over time.

A secondary set of analyses on the same outcomes using a traditional least squares general linear model (GLM) approach was done to corroborate the REML results. In these GLM analyses, study data were analyzed for the study-completing sample only $(N=74)$. The completer sample provides an estimate of the benefits of MBSR among those who completed all study assessments (Kazdin, 2003). All continuous predictor variables were centered before GLM analyses (Aiken \& West, 1991). Homogeneity of variance and variance differences (latter assessed by the Mauchly test of sphericity) were checked in all GLM analyses, and the Huynh-Feldt epsilon correction was applied where indicated.

Before beginning these ITT and completer sample analyses, preliminary multivariate analysis of variance (MANOVA) of the SCL-90-R was done to determine whether results differed across the six subscale scores used. This initial analysis was performed to control for experiment-wise error arising from analysis of highly correlated subscale scores. The MANOVA on SCL-90-R scores revealed a significant Condition $\times$ Subscale interaction $(p<.05)$ and a significant Condition $\times$ Time $\times$ Subscale interaction $(p<$ .02). This significant variance in condition and Condition $\times$ Time effects between the subscales supported analyses testing the effects of the Condition $\times$ Treatment interaction on each SCL-90-R subscale score separately.

Where the Condition $\times$ Time effect was significant in the REML and GLM analyses, post hoc $t$ tests were used to examine treatment condition differences that occurred from pretest to posttest and pretest to follow-up. Treatment condition differences at each of the posttest and follow-up time points were also tested.

A third type of analysis, namely least-squares multiple regression, examined the effects of treatment condition on diagnostic change over time while controlling for the effects of relevant demographic and clinical variables. This analysis was limited to the completer sample.

For all three types of analyses, effect size estimates were calculated using Cohen's $d$ estimates where appropriate to examine the clinical significance of the observed changes. For the mixed model and GLM results, this was done using standardized mean pretest-follow-up differences between MBSR and TAU groups. For the multiple regression results, standardized regression weights are reported.

The fourth primary set of analyses assessed whether the mental health changes observed through MBSR (relative to TAU) participation were reliable. Reliable change refers to the extent to which changes in scaled instrument scores between measurement points exceed what could be attributed to measurement error of the instruments (e.g., Evans, Margison, \& Barkham, 1998). This assessment has been used extensively in therapeutic contexts (e.g., Jacobson, Roberts, Berns, \& McGlinchey, 1999). The reliable change in scaled outcome scores from preintervention to follow-up was assessed using the now classic method proposed by Jacobson and Truax (1991). In this approach, measurement error-the standard error that accounts for the two pretest to posttest measurements made (or preintervention and follow-up measurements made), symbolized as $S E_{\text {diff }}$-is primarily a function of the initial standard deviation of the scale and its internal consistency, as follows: $S D_{1} \sqrt{ } 2 \sqrt{ }(1-\alpha)$. A reliable change index $(\mathrm{RCI})$ for each 
scale was computed by subtracting the follow-up score from the pretest score then dividing by its associated $S E_{\text {diff }}$ term. Individual RCI scores exceeding 1.96 (improvement) or -1.96 (worsening) were considered to reflect reliable change, $p<.05$.

Before beginning analyses, all continuous variables were checked for skewness and kurtosis and converted to categorical scores where necessary. Specifically, given that dosages of the various medications were quite positively skewed (with many taking no medication), a single, dichotomized (yes $/$ no ) medicationuse variable was created for each time point. Hospital and group therapy visits were also positively skewed, so for each time interval they were dichotomized (yes/no). Given the low prevalence of individual disorders other than mood and anxiety disorders, an other diagnoses category was created to capture these. These other disorders included substance-related disorders and disruptive behavior disorders (e.g., attention-deficit/hyperactivity disorder). $\mathrm{V}$-code diagnoses (relational problems) were coded as 0,1 , and 2 or more codes. Finally, the SCL-90 subscale scores were nonnormal in both treatment conditions at one or more time points due to wide score ranges (see Table 3 and appendix). However, inspection of the mixed model and GLM residual plots revealed little or no deviations from normality. Because these analyses are quite robust with nonnormal data, the original scaling of the SCL-90 scores was retained.

\section{Results}

\section{Patient Characteristics}

As a whole, the ITT sample was predominantly female (73.5\%), and most participants were Caucasian $(45.1 \%)$ or Hispanic/ Latino(a) (28.4\%). The remainder were Asian (5.9\%), African American $(2.9 \%)$, Native American (1\%) or of mixed racial or ethnic descent $(16.7 \%)$. The average age of participants was 15.35 years of age $(S D=1.20$, range $=14$ to 18 years $)$. All disorders in the sample were based on $D S M-I V-T R$ Axis I diagnoses; the most common were mood disorders (49\%) and anxiety disorders
(30.4\%). Other disorders were found in $24.5 \%$ of the sample. A majority of individuals in the sample $(56.9 \%)$ were also diagnosed with V-codes (e.g., parent-child relational problems and/or problems related to abuse or neglect). There was considerable comorbidity in the sample.

Baseline demographic, diagnostic, and pretest psychotherapeutic characteristics of the sample are displayed in Table 1. As shown there, the MBSR and TAU groups did not significantly differ on baseline demographic characteristics, with one exception: MBSR participants were slightly older than those receiving TAU (by approximately 8 months, $p<.01$ ). The treatment groups did not differ on diagnostic or therapeutic characteristics except on the higher incidence of other Axis I diagnoses among MBSR participants, $p<.05$. Given these differences, age and incidence of other diagnoses were included in preliminary outcome analyses; however, neither variable predicted any of the outcomes, so they were dropped from the main analyses presented here. In the completer sample, the 39 patients who completed the MBSR program were compared with the 11 who did not; no significant differences between these two groups were found on any of the baseline characteristics (all $p \mathrm{~s}>.05$ ).

\section{TAU Received}

Mental health treatment received by patients in both the TAU and MBSR conditions was monitored at each of the three assessment points and is summarized in Table 2 for the intent-to-treat sample. There were no significant differences between the TAU and MBSR conditions for any of the measures of treatment received at each assessment point (all $p \mathrm{~s}>.10$ ).

\section{Treatment Effects on Self-Reported Mental Health Outcomes}

Preliminary $t$ tests revealed no differences between conditions in self-reported mental health at baseline. Preliminary analyses

Table 1

Demographic and DSM-IV-TR Axis I Diagnostic Characteristics According to Treatment Group

\begin{tabular}{|c|c|c|c|c|c|}
\hline \multirow[b]{2}{*}{ Variable } & \multicolumn{2}{|c|}{$\operatorname{MBSR}(n=50)$} & \multicolumn{2}{|c|}{ TAU $(n=52)$} & \multirow[b]{2}{*}{$p_{\text {diff }}$} \\
\hline & $N$ & $\%$ & $N$ & $\%$ & \\
\hline \multicolumn{6}{|c|}{ Demographic characteristics } \\
\hline Gender (female) & 35 & 70.0 & 40 & 76.9 & .42 \\
\hline Age $(M \pm S D)$ & $15.7 \pm 1.13$ & & $15.0 \pm 1.19$ & & .006 \\
\hline Ethnicity/Race & & & & & .11 \\
\hline Caucasian & 19 & 38.0 & 27 & 51.9 & \\
\hline Hispanic/Latino & 19 & 38.0 & 10 & 19.2 & \\
\hline African American & 1 & 2.0 & 2 & 3.8 & \\
\hline Mixed & 6 & 12.0 & 11 & 21.2 & \\
\hline Other & 5 & 10.0 & 2 & 3.8 & \\
\hline \multicolumn{6}{|l|}{ Diagnostic characteristics } \\
\hline Mood disorder & 27 & 54.0 & 23 & 44.2 & .32 \\
\hline Anxiety disorder & 17 & 34.0 & 14 & 26.9 & .44 \\
\hline V-code disorder & 29 & 58.0 & 29 & 55.8 & .32 \\
\hline Other disorder & 17 & 34.0 & 8 & 15.4 & .03 \\
\hline
\end{tabular}

Note. $\quad N=102$. The $p_{\text {diff }}$ column shows significance levels based on $t$ and $\chi^{2}$ tests of group differences. MBSR $=$ mindfulness-based stress reduction; TAU = treatment as usual. 
Table 2

Treatment Received From Other Sources by Participants in MBSR and TAU Groups in Intent-toTreat Sample at Pretreatment, Posttreatment, and Follow-Up Time Points

\begin{tabular}{|c|c|c|c|c|c|}
\hline \multirow[b]{2}{*}{ Variable } & \multicolumn{2}{|c|}{ MBSR } & \multicolumn{2}{|c|}{ TAU } & \multirow[b]{2}{*}{$p_{\text {diff }}$} \\
\hline & $N$ & $\%$ & $N$ & $\%$ & \\
\hline \multicolumn{6}{|c|}{ Psychotherapeutic treatment } \\
\hline \multicolumn{6}{|c|}{ Individual therapy } \\
\hline Pretest & 50 & 100.0 & 52 & 100.0 & .18 \\
\hline Posttest & 33 & 84.6 & 31 & 64.7 & .35 \\
\hline Follow-up & 20 & 58.8 & 21 & 52.5 & .78 \\
\hline \multicolumn{6}{|l|}{ Group therapy } \\
\hline Pretest & 23 & 46.0 & 22 & 42.3 & .71 \\
\hline Posttest & 2 & 5.1 & 6 & 13.0 & .21 \\
\hline Follow-up & 3 & 8.8 & 4 & 10.0 & .86 \\
\hline \multicolumn{6}{|c|}{ Psychotropic medication } \\
\hline Pretest & 25 & 50.0 & 20 & 38.5 & .24 \\
\hline Posttest & 18 & 46.2 & 22 & 47.8 & .88 \\
\hline Follow-up & 15 & 44.1 & 19 & 47.5 & .77 \\
\hline \multicolumn{6}{|c|}{ Psychiatric hospitalization } \\
\hline Pretest & 8 & 16.0 & 4 & 7.7 & .19 \\
\hline Posttest & 0 & 0.0 & 3 & 6.5 & .10 \\
\hline Follow-up & 1 & 2.9 & 1 & 2.5 & .91 \\
\hline
\end{tabular}

Note. The $p_{\text {diff }}$ column shows significance levels based on $t$ tests (number of individual therapy sessions) and $\chi^{2}$ tests of group differences. MBSR $=$ mindfulness-based stress reduction; TAU $=$ treatment as usual; ITT $=$ intent-to-treat sample. MBSR $n \mathrm{~s}=50,39$, and 34 at pretest, posttest and follow-up, respectively; TAU $n \mathrm{~s}=52$, 46 , and 40 at pretest, posttest, and follow-up, respectively.

showed that gender and the presence of Axis I mood disorder at baseline were significantly related to several self-report outcomes. Age, number of prestudy hospitalizations, and presence of a V-code diagnosis were also related to several of these outcomes. These variables were included as predictors in preliminary analy- ses where relevant. If significant, either as main effects or in interaction with time, the variables were retained for the reported analyses.

Table 3 shows the REML results for all continuous self-reported mental health outcome measures. Relative to TAU controls, ITT

Table 3

Mean (and SD) Values in MBSR and TAU Groups At Pretreatment, Posttreatment, and Follow-Up Time Points for Intent-to-Treat Sample

\begin{tabular}{|c|c|c|c|c|c|c|c|c|c|}
\hline \multirow[b]{2}{*}{ Variable } & \multicolumn{4}{|c|}{ MBSR } & \multicolumn{4}{|c|}{ TAU } & \multirow[b]{2}{*}{$p_{\text {inter }}$} \\
\hline & Pretest & Posttest & Follow-up & $d$ & Pretest & Posttest & Follow-up & $d$ & \\
\hline \multicolumn{10}{|l|}{ Self-report measures } \\
\hline STAI-present & $3.81(1.04)$ & $3.07(1.01)$ & $2.94(1.40)$ & 0.70 & $3.53(1.15)$ & $3.11(1.26)$ & $3.54(1.35)$ & -0.01 & .005 \\
\hline STAI-past & $4.29(0.93)$ & $3.35(1.09)$ & $3.36(1.39)$ & 0.79 & $4.01(1.28)$ & $3.53(1.35)$ & $3.82(1.46)$ & 0.14 & .04 \\
\hline PSS & $24.12(5.73)$ & $19.46(6.01)$ & $18.68(6.54)$ & 0.89 & $21.81(7.11)$ & $19.80(6.05)$ & $20.63(7.51)$ & 0.16 & .02 \\
\hline$S E$ & $18.88(5.71)$ & $15.80(5.30)$ & $15.44(5.89)$ & 0.59 & $17.04(7.00)$ & $17.80(7.14)$ & $18.03(6.51)$ & -0.15 & .0001 \\
\hline Sleep quality & & $4.82(1.23)$ & $4.65(1.25)$ & 0.14 & & $4.56(1.14)$ & $3.56(1.16)$ & 0.87 & .02 \\
\hline \multicolumn{10}{|l|}{ SCL-90 ${ }^{\mathrm{a}}$} \\
\hline Somatization & $15.82(10.41)$ & $10.62(8.82)$ & $8.47(7.86)$ & 0.80 & $14.02(11.11)$ & $13.59(11.59)$ & $15.35(13.09)$ & -0.11 & .0008 \\
\hline Obsessive & $20.24(8.05)$ & $13.03(8.42)$ & $11.47(7.75)$ & 1.11 & $17.44(10.79)$ & $14.56(10.85)$ & $15.50(10.60)$ & 0.18 & .0006 \\
\hline Interpersonal & $14.10(8.16)$ & $9.56(7.33)$ & $7.88(6.92)$ & 0.82 & $15.12(9.36)$ & $13.35(9.05)$ & $13.23(9.31)$ & 0.20 & .03 \\
\hline Depression & $22.74(11.84)$ & $13.44(9.66)$ & $12.21(10.37)$ & 0.95 & $22.48(12.96)$ & $19.30(13.36)$ & $18.58(12.29)$ & 0.31 & .001 \\
\hline Anxiety & $13.80(9.41)$ & $8.80(7.51)$ & $7.85(8.53)$ & 0.66 & $13.62(10.06)$ & $10.89(10.73)$ & $11.90(10.65)$ & 0.17 & .60 \\
\hline Hostility & $9.94(6.26)$ & $6.87(5.13)$ & $5.94(5.58)$ & 0.67 & $10.02(6.80)$ & $8.39(6.58)$ & $8.48(6.21)$ & 0.24 & .12 \\
\hline \multicolumn{10}{|l|}{ Clinical measures } \\
\hline Diagnostic change $n(\%)$ & & $19(48.7)$ & $12(30.8)$ & & & $0(0.0)$ & $1(2.2)$ & & \\
\hline $\mathrm{GAF}$ & $59.54(6.51)$ & $63.67(6.59)$ & $66.33(6.78)$ & -1.02 & $63.81(8.13)$ & $62.48(8.24)$ & $61.76(9.00)$ & 0.24 & .0001 \\
\hline
\end{tabular}

Note. $\quad N=102$ at pretest. MBSR $=$ mindfulness-based stress reduction; TAU $=$ treatment as usual; STAI-present $=$ State-Trait Anxiety Inventory, state; STAI-past $=$ State-Trait Anxiety Inventory, trait; PSS = Perceived Stress Scale; SE = Rosenberg Self-Esteem; Sleep quality = sleep quality change; SCL-90 = Symptom Checklist-90-R. Sleep quality change and diagnostic change at posttest are in reference to pretest, and at follow-up are in reference to posttest. The $d$ column shows Cohen's $d$ effect sizes based on unadjusted pretest and follow-up means within each group. The $p_{\text {inter }}$ column shows significance levels of restricted maximum likelihood mixed model Treatment Condition $\times$ Time interaction.

${ }^{a}$ The SCL-90 subscales were nonnormally distributed in each treatment condition at one or more time points due to wide score ranges; median and range (minimum and maximum) values are included in the appendix. 
sample MBSR participants showed significant improvements over time in state and trait anxiety, perceived stress, self-esteem, and four of the six indicators of psychopathology assessed-namely, somatic, obsessive-compulsive, interpersonal sensitivity, and depressive symptoms (all $p \mathrm{~s}<.05$ ). There were no Condition $\times$ Time effects on psychopathological symptoms of anxiety and hostility (both $p s>$.17). There was a Condition $\times$ Time effect on change in sleep quality $(p<.05)$, but this effect was due to deterioration in sleep in the TAU group. Indication of the magnitude of treatment effects that is independent of sample size is provided by effect size estimates, using Cohen's $d$, based on pretest to follow-up changes in group means. As Table 3 shows, the effect sizes associated with MBSR participation on the statistically significant mental health outcomes were consistently large in this ITT sample.

Post hoc tests showed that significant $(p<.05)$ or trending toward significant $(p<.08)$ improvements in MBSR relative to control participants occurred between pretest and posttest time points on all of the outcomes that showed significant change in the primary analyses. Significant improvements in MBSR participants relative to controls were found on all of these outcomes between pretest and follow-up points (all $p \mathrm{~s}<.05$ ).

Post hoc analyses ( $t$ tests) were also used to examine group differences at the posttest and follow-up time points separately. At posttest, group differences in self-report scores were in the direction favoring MBSR participants but were generally not statistically significant (most $p s>.05$ ) and in the small to medium range of effect sizes, as indexed by Cohen's $d\left(M_{d}=0.40 ;\right.$ range $_{d}=0.15$ to 0.79 across variables). At follow-up, group differences in outcomes favoring MBSR participants were generally significant $(p s<.05)$ or trending toward significant $(p s<.10)$ across variables and in the medium to large effect size range $\left(M_{d}=0.51\right.$; range $_{d}=0.28$ to 0.92 ).

\section{Other Findings}

Main effects for gender and mood disorder were found in several of the analyses. Girls showed higher SCL-90-R depressive symptoms across time $(p<.05)$. Baseline mood disorder was related to higher state and trait anxiety, perceived stress, and SCL-90-R somatic and depressive symptoms over time (all $p \mathrm{~s}<$ $.05)$. The presence of other disorders interacted with time to predict interpersonal sensitivity symptoms $(p<.05)$; the form of the interaction showed a waning relation of disorder to this outcome over the three time points. No other significant main or interaction effects were found.

Results of study-completing sample GLM analyses of the selfreported outcomes closely paralleled those of the ITT analyses reported here. All significant Treatment Condition $\times$ Time effects reported already were also significant in the completer sample, though most at a slightly lower level of significance. The same main effects for gender and mood disorder reported already were also found in these GLM analyses.

\section{Treatment Effects on Clinical Mental Health Outcomes}

GAF analyses. Preliminary analyses revealed that treatment condition patients had lower GAF scores than controls at baseline $(M=59.54$ vs. $M=63.81$, respectively, $p<.01)$. Thus, within- group REML analyses were used to check the accuracy of the mixed REML analyses on this outcome. The use of post hoc tests (see Statistical Analyses section, above) also helped to circumvent the potential bias in the results arising from this baseline difference. Preliminary analyses also showed that gender and the presence of Axis 1 mood disorder at baseline were significantly related to GAF scores. If either the main effect or the interaction with time effect was significant in models that included the treatment condition main effect, the gender and mood disorder variables were retained for the analyses to be reported here.

The bottom of Table 3 shows the mixed REML results for GAF score in the ITT sample. Compared with TAU controls, MBSR participants showed strongly significant improvements over time in GAF score, and the effect size was large. This finding was confirmed by follow-up within-group REML models (MBSR improvement, $p<.0001$; TAU decline, $p<.05$ ). Post hoc tests showed that significant improvements in GAF score in MBSR relative to TAU participants occurred between pretest and posttest time points $(p<.0001)$ and between pretest and follow-up points $(p<.0001)$. Interestingly, GAF scores in MBSR participants relative to controls continued to improve from posttest to the follow-up point, $p<.005$.

Post hoc analyses also examined group differences in GAF scores at post-test and follow-up separately. A $t$ test of this group difference at posttest was nonsignificant $(p>.12)$, and the effect size was small $(d=.13)$. At follow-up, the group difference in GAF score favoring MBSR participants was significant $(p<.001)$ and moderate in effect size $(d=.56)$.

Other GAF findings. Main effects for gender and mood disorder were also found in the primary GAF model. Girls showed higher GAF scores across time, $p<.01$. Baseline mood disorder was related to lower GAF scores across time, $p<.01$. No other main or interaction effects on GAF score were found.

In the study-completing sample, GLM analyses again found that MBSR participants showed highly significant improvement in GAF scores relative to TAU controls $(p<.0001)$. The MBSR participant improvements were found between pretest and posttest, and between pretest and follow-up (both $p \mathrm{~s}<.05$ ).

Diagnostic change analyses. To test the effect of treatment on diagnostic change over the duration of the study, preliminary parametric ( $t$ test) and nonparametric (chi-square) analyses were conducted to select appropriate control variables for the main regression analysis. Several demographic and clinical variables discriminated between participants who showed diagnostic improvement or not from pretest to follow-up: gender; presence of mood disorder; V-code diagnoses; other diagnoses; number of individual mental health visits prior to, during, and following the 8-week pretest-posttest portion of the study; number of in-patient hospitalizations prior to the study; and use of psychotropic medication at baseline (all $p \mathrm{~s}<.05$ ). These control variables, along with treatment condition, were included in a standard multiple regression analysis of prediction of diagnostic change from pretest to follow-up in the completer sample.

The regression model was significant, $F(10,84)=8.76, p<$ $.0001, R^{2}=.54$. Relative to controls, MBSR participants were much more likely to show diagnostic improvement over the course of the study, $\beta=.57, p<.0001$. Specifically, $54 \%(n=21)$ of MBSR participants in the completer sample showed diagnostic improvement, whereas only $1(2.2 \%)$ receiving TAU did so (see 
lower portion of Table 3). Chi-square analysis of group differences in diagnostic change was highly significant at both posttest $(p<$ $.0001)$ and follow-up $(p<.001)$.

This difference in diagnostic picture appeared across most of the diagnostic categories examined here. In the MBSR condition, the pretest and follow-up prevalence rates were $58.8 \%$ and $26.5 \%$, respectively, for mood disorder; $35.3 \%$ and $20.6 \%$ for anxiety disorder; and $35.3 \%$ and $14.7 \%$ for other diagnoses. Prevalence of two or more $\mathrm{V}$-codes dropped from $23.5 \%$ to $14.7 \%$ from pretest to follow-up. In the TAU condition, prevalence rates at these two time points were $47.5 \%$ and $57.5 \%$ for mood disorder; $27.5 \%$ and $37.5 \%$ for anxiety disorder; $12.7 \%$ and $22.5 \%$ for other diagnoses; and $10 \%$ and $12.7 \%$ with two or more V-code diagnoses. Frequency analyses of the group differences in specific diagnoses at follow-up showed that MBSR participants evidenced a significantly lower rate of mood disorder, $\chi^{2}(1, N=74)=7.21, p<.01$. The groups did not differ significantly in the other diagnostic frequencies at the follow-up point.

Other diagnostic change findings. In this completer sample, boys were more likely to show diagnostic improvement over the course of the study than girls, $\beta=.25, p<.01$. No other main effects were found.

\section{Reliability of Treatment Effects on Self-Reported Mental Health Outcomes}

MBSR had statistically significant and sizable effects on many of the mental health outcomes examined in this study. Still unknown is whether the MBSR intervention produced reliable changes relative to TAU. In the present context, this issue is most relevant to the selfreported outcomes, where measurement error may render individual changes less reliable. To determine whether participants in the MBSR group, relative to TAU participants, showed reliable changes on the self-report measures, study-completing participants were classified as having shown reliable improvements, declines, or no changes from pretest to follow-up using the RCI.

Table 4 shows the RCI results-specifically, the number of individuals who showed significant increases, decreases, or no significant changes on each self-report scale. To a degree, these results complement those of the statistical analyses in highlighting the discrepancy between MBSR and TAU conditions in the patterns of mental health change across the duration of the study. For example, more than $50 \%$ of participants in the MBSR condition showed a reliable increase in STAI present anxiety compared with $20 \%$ of TAU participants. Table 4 also shows that rates of reliable improvement versus no change or worsening differed significantly between treatment groups in present anxiety, self-esteem, and SCL somatization and obsessive-compulsiveness (all $p s<.05$ ) and differed at a trend toward significant level in perceived stress, $p<$ .07. Group differences in reliable change on the other scaled variables were not significant, but almost all were in the expected direction.

\section{Mindfulness Practice Effects on Mental Health Outcomes}

Exploratory REML mixed model analyses tested the predictive role of the frequency and duration of each of the four practices taught in the MBSR course on changes in the self-report and clinical measures that showed significant Condition $\times$ Time interactions in the primary REML models with the ITT sample. For these analyses, each of the measures of practice during the course was aggregated across weeks, and their effects were tested after entry of the same control variables included in the previously presented models.

Given the large number of tests performed, only results pertaining to sitting meditation frequency and duration are presented, as these measures showed the most frequent effects on mental health change. For example, more days of sitting practice predicted an increase in GAF score and declines in SCL-90 depressive symptoms (and anxiety) from baseline to follow-up, all $p \mathrm{~s}<.05$. More days of sitting practice also marginally predicted more diagnostic change, $p<.08$. In separate REML analyses, average length (in minutes) of sitting practice sessions per week during the course predicted a greater increase in GAF score $(p<.005)$ and sleep quality $(p<.05)$ and a greater decline in SCL-90 depressive (and anxiety) symptoms $(p \mathrm{~s}<.05)$ from baseline to follow-up. In the

Table 4

Summary of Results Showing Number (and \%) of Study-Completing Participants in MBSR and TAU Groups With Significant Reliable Change Index Scores

\begin{tabular}{|c|c|c|c|c|c|c|c|c|}
\hline \multirow[b]{2}{*}{ Scale } & \multicolumn{3}{|c|}{ MBSR } & \multicolumn{3}{|c|}{ TAU } & \multirow[b]{2}{*}{$x^{2}$} & \multirow[b]{2}{*}{$p$} \\
\hline & Worsened & No change & Improved & Worsened & No change & Improved & & \\
\hline STAI-present & $2(5.9)$ & $14(41.2)$ & $18(52.9)$ & $5(12.5)$ & $27(67.5)$ & $8(20.0)$ & 8.75 & .003 \\
\hline STAI-past & $3(8.8)$ & $12(35.3)$ & $19(55.9)$ & $5(12.5)$ & $19(47.5)$ & $16(40.0)$ & 1.86 & .17 \\
\hline PSS & $2(5.9)$ & $22(64.7)$ & $10(29.4)$ & $2(5.0)$ & $33(82.5)$ & $5(12.5)$ & 3.25 & .07 \\
\hline$S E$ & $1(2.9)$ & $21(61.8)$ & $12(35.3)$ & $6(15.0)$ & $28(70.0)$ & $6(15.0)$ & 4.11 & .04 \\
\hline \multicolumn{9}{|l|}{ SCL-90 } \\
\hline Somatization & $0(0.0)$ & $22(64.7)$ & $11(32.4)$ & $5(12.5)$ & $30(75.0)$ & $5(12.5)$ & 4.27 & .04 \\
\hline Obsessive & $0(0.0)$ & $20(58.8)$ & $14(41.2)$ & $2(5.0)$ & $31(77.5)$ & $7(17.5)$ & 5.07 & .02 \\
\hline Interpersonal & $0(0.0)$ & $25(73.5)$ & $9(26.5)$ & $4(10.0)$ & $25(62.5)$ & $11(27.5)$ & 0.01 & .92 \\
\hline Depression & $0(0.0)$ & $20(58.8)$ & $14(41.2)$ & $4(10.0)$ & $25(62.5)$ & $11(27.5)$ & 1.54 & .22 \\
\hline Anxiety & $1(2.9)$ & $25(73.5)$ & $8(23.5)$ & $1(2.5)$ & $31(77.5)$ & $8(20.0)$ & 0.14 & .71 \\
\hline Hostility & $1(2.9)$ & $26(76.5)$ & 7 (20.6) & $4(10.0)$ & $27(67.5)$ & $9(22.5)$ & 0.04 & .84 \\
\hline
\end{tabular}

Note. $\quad$ MBSR $=$ mindfulness-based stress reduction; TAU $=$ treatment as usual; STAI-present $=$ State-Trait Anxiety Inventory, state; STAI-past $=$ State-Trait Anxiety Inventory, trait; PSS = Perceived Stress Scale; SE = Rosenberg Self-Esteem; SCL-90 = Symptom Checklist-90-R. Chi-square tests compare rates of improvement versus no change/worsened across MBSR and TAU groups. 
completer sample analyses, GLM results were very similar. Significant effects for body scan, yoga, and informal practice were also found, though considerably fewer in number than for sitting practice. Though provocative, these results must be interpreted with caution, given the purely exploratory nature of the analyses.

\section{MBSR Participant Treatment Evaluations}

On postintervention program evaluation forms, all who completed the MBSR program indicated that they had obtained something of lasting value and rated the personal importance of the program moderately highly $(M=5.26, S D=.91$, on a 7-point scale). Ninety-five percent of MBSR participants made openended comments, all of which were positive; these highlighted treatment-related changes in stress, medication usage, and sleep, and beneficial effects of mindfulness practice. No suggestions for MBSR program improvement were made.

\section{Discussion}

A growing body of research suggests that mindfulness-based psychosocial interventions are effective for a wide range of mental and physical health disorders in adult populations (e.g., Baer, 2003; Brown et al., 2007; Grossman et al., 2004). Yet little is known about the effects of such interventions for adolescents. The present study is the first randomized clinical trial known to us that was designed to assess the effectiveness of a mindfulness-based intervention for adolescents in an outpatient psychiatric facility. Results from this randomized clinical trial provide evidence that one such intervention, MBSR, may be beneficial as an adjunct to outpatient mental health treatment for adolescents with heterogeneous mental health diagnoses.

Results of the study indicated that in both completer and intentto-treat samples, MBSR + TAU participation significantly reduced self-reported anxiety, depressive, and somatization symptoms, and improved self-esteem and sleep quality compared with TAU-only control participants. In the study-completing MBSR group, there were also significant declines in self-reported perceived stress, obsessive symptoms, and interpersonal problems relative to TAU controls. Also in both samples, MBSR participants showed significant increases in GAF scores and a higher percentage of mental health changes than TAU controls, which were large enough to warrant a change in number of diagnoses. Across samples, more than $45 \%$ of the MBSR group showed such diagnostic change, whereas almost none of the TAU control participants did so. Such change was most pronounced among MBSR participants with mood disorders, where the prevalence at follow-up was less than half that seen at pretest in both samples. Nonsignificant declines in prevalence rates over this time period in the MBSR group were also seen in the other diagnostic categories included in the study, namely anxiety disorders, V-code diagnoses, and other disorders, including substance-related and disruptive disorders. In contrast, the TAU group showed very few declines or showed increases in prevalence in these disorders over the pretest to follow-up study period in both ITT and completer samples.

These results were obtained on the basis of changes in mental health from pretest through to 3 months after completion of the MBSR program. Most statistical effect sizes of MBSR were large in the ITT and completer samples (the latter generally not reported here). Where significant statistical effects of treatment were found on the scaled (self-reported) outcomes, reliable improvements across these measures were found in $32 \%-53 \%$ of the studycompleting MBSR group, compared with $13 \%-20 \%$ of the control group. These reliable change findings suggest that the self-reported changes were not attributable to measurement error variance.

At-home mindfulness practice is central to the MBSR program, and our exploratory analyses in the completer sample found that amount of formal practice, particularly number of days of sitting mindfulness practice and average length of each practice session, were related to a number of changes from baseline to follow-up in the clinical or self-report measures assessed in the study. Previous research (e.g., Carson, Carson, Gil, \& Baucom, 2004; Davidson et al., 2003; Shapiro et al., 2007) has been inconsistent on the topic of mindfulness practice effects, but it is important for future research to continue to explore the value of these practicesmeasured in terms of both quantity and quality of time spent in them-to intervention outcomes, particularly as mindfulness practice is a potentially key mechanism that may explain the effects of MBSR

In general, the present randomized trial suggested that MBSR is well tolerated by adolescents with various mental health problems. Seventy-eight percent of participants randomized to MBSR completed the intervention, which compares favorably to completion benchmarks in cognitive-behavioral therapy efficacy trials for children and adolescents (above 75\%; Hunsley \& Lee, 2007). The present MBSR completion rate is also similar to the average rate observed in adult MBSR trials (Baer, 2003). The fact that very similar results on GAF and self-reported outcomes were found in both ITT and completer samples in this study suggests that nonadherence to the protocol (study dropout) was not related to the outcomes. The reductions in self-reported psychological symptomatology observed in both ITT and completer samples in this study are consistent with findings from MBSR research conducted with adult populations (e.g., Baer, 2003; Grossman et al., 2004). Little research has examined the efficacy of MBSR and related mindfulness interventions with adolescents, and much of the research conducted to date has been preliminary, based on small samples and correlational designs (e.g., Bootzin \& Stevens, 2005). The present randomized trial suggests that MBSR may have positive effects on both self-reported psychological experience and clinically significant outcomes that can be sustained over time.

\section{Limitations and Future Research}

Given the novelty of this area of inquiry, more research is needed to assess the replicability and generalizability of these findings. The present results must be considered specific to the population under study, namely, a heterogeneous population of adolescent psychiatric outpatients, most of who were diagnosed with mood and anxiety disorders. Examining the effects of this intervention with a homogeneous population of adolescents with single primary diagnoses will shed light on the diagnostic specificity of the expectable benefit from this mindfulness-based intervention.

Further, the sample was largely female, and although the present research, in line with past research, did not uncover gender differences in responsiveness to MBSR, research using samples with more balanced gender compositions is needed to more adequately 
test the benefits of this program for adolescent boys and girls. Other limitations of the study included the use of a wait-list control group and the relatively brief (3-month) follow-up period. Research using one or more active control groups will help to ameliorate concerns about potential confounding effects of differential motivation and attention between the treatment and wait-list control groups. The use of longer post-intervention follow-up periods will be helpful to further assess the endurance of treatment effects. In this study clinical diagnoses were assigned in accord with standard practice and training; but without the use of a standardized interview or multiple clinician ratings, there was no means to assess diagnostic reliability. This is a clear limitation, but it was ameliorated to a degree by the fact that clinical assessors were naïve to participants' treatment condition and were not aware that clinical ratings would be used for research purposes, so there was no basis to expect differential diagnostic change or GAF scoring according to condition. However, future research should ensure that reliability of clinical measures can be assessed.

Additionally, the study assessed a broad range of indicators of mental health, and no adjustments to statistical significance levels were made. The decision to accept the standard $(p<.05)$ level of significance was made because no prespecified predictions about treatment effects on specific outcomes were made. Rather, the study had general hypotheses about mental health effects, and because the intervention (MBSR) had never been conducted with an adolescent population in a randomized trial, it was considered important to test the effects of the intervention in a reasonably liberal fashion. With the present research showing strongest effects in domains of perceived stress, self-esteem, anxiety, depressive symptoms, and global adaptive functioning, future research will be better prepared to narrow the field of investigation of MBSR effects, formulating hypotheses regarding specific benefits of the intervention.

It might be argued that the outcomes chosen in this study were most relevant to psychological processes targeted by MBSR; but the assessment of wide range of mental health indicators argues against this limitation. Indeed, these results raise the question as to whether MBSR is a specific treatment for specific symptoms or disorders or may be broadly applicable to a range of psychological symptoms or populations. Research with adolescents has found modest treatment specificity effects (e.g., Kolko, Brent, Baugher, Bridge, \& Birmaher, 2000), but addressing this issue with respect to MBSR will clearly require further research.

It is also possible that those receiving MBSR were biased to report greater improvement on the self-report measures due to the greater attention they received. This is less likely to explain clinician-rated treatment differences, however, and does not well explain why treatment differences on both self- and clinicianreported outcomes continued to obtain at 3 months postintervention. Yet in future research, use of an active control condition along with assessment of treatment benefit expectancy would help to test this alternative explanation for MBSR effects. Last, it is possible that those in the MBSR group received more total treatment time than those in TAU group, which may have helped to account for some of the MBSR group improvements. Future research should control for concurrent mental health treatment time in assessing MBSR effects.

\section{References}

Aiken, L. S., \& West, S. G. (1991). Multiple regression: Testing and interpreting interactions. Newbury Park, CA: Sage.

American Psychiatric Association. (2000). Diagnostic and statistical manual of mental disorders (4th ed.). Washington, DC: Author.

Baer, R. A. (2003). Mindfulness training as a clinical intervention: A conceptual and empirical review. Clinical Psychology: Science and Practice, 10, 125-143.

Barnes, V. A., Davis, H. C., Murzynowski, J. B., \& Treiber, F. A. (2004). Impact of meditation on resting and ambulatory blood pressure and heart rate in youth. Psychosomatic Medicine, 66, 909-914.

Biegel, G. M. (2005). Mindfulness-based stress reduction for adolescents. Unpublished manuscript.

Boden, J. M., Fergusson, D. M., \& Horwood, L. J. (2008). Does adolescent self-esteem predict later life outcomes? A test of the causal role of self-esteem. Development and Psychopathology, 20, 319-339.

Bootzin, R. R., \& Stevens, S. J. (2005). Adolescents, substance abuse, and the treatment of insomnia and daytime sleepiness. Clinical Psychology Review, 25, 629-644.

Brown, K. W., \& Ryan, R. M. (2003). The benefits of being present: Mindfulness and its role in psychological well-being. Journal of Personality and Social Psychology, 84, 822-848.

Brown, K. W., Ryan, R. M., \& Creswell, J. D. (2007). Mindfulness: Theoretical foundations and evidence for its salutary effects. Psychological Inquiry, 18, 211-237.

Bryk, A. S. \& Raudenbush, S. W. (1992). Hierarchical linear models: Applications and data analysis methods. Newbury Park, NJ: Sage.

Carson, J. W., Carson, K. M., Gil, K. M., \& Baucom D. H. (2004). Mindfulness-based relationship enhancement. Behavior Therapy, 35, 471-494.

Cohen, S., \& Williamson, G. M. (1988). Perceived stress in a probability sample in the United States. In S. Spacapan \& S. Oskamp (Eds.), The social psychology of health (pp. 31-67). Beverly Hills, CA: Sage.

Collishaw, S., Maughan, B., Goodman, R., \& Pickles, A. (2004). Time trends in adolescent mental health. Journal of Child Psychology and Psychiatry, 45, 1350-1362.

Davidson, R. J., Kabat-Zinn, J., Schumacher, J., Rosenkranz, M., Muller, D., Santorelli, S. F., et al. (2003). Alterations in brain and immune function produced by mindfulness meditation. Psychosomatic Medicine, 65, 564-570.

Derogatis, L. (1977). The SCL-90-R: Administration, scoring and procedures manual. Baltimore: Clinical Psychometric Research.

Emslie, G. J., Mayes, T. L., Laptook, R. S., \& Batt, M. (2003). Predictors of response to treatment in children and adolescents with mood disorders. Psychiatric Clinics of North America, 26, 435-456.

Evans, C., Margison, F., \& Barkham, M. (1998). The contribution of reliable and clinically significant change methods to evidence-based mental health. Evidence Based Mental Health, 1, 70-72.

Frydenberg, E., Lewis, R., Bugalski, K., Cotta, A., McCarthy, C., Luscombe-Smith, N., et. al. (2004). Prevention is better than cure: Coping skills training for adolescents in school. Educational Psychology in Practice, 20, 117-134.

Garcia, J. A., \& Weisz, J. R. (2002). When youth mental health care stops: Therapeutic relationship problems and other reasons for ending youth outpatient treatment. Journal of Consulting and Clinical Psychology, 70, $439-443$

Greene, J. W., \& Walker, L. S. (1997). Psychosomatic problems and stress in adolescence. Pediatric Clinics of North America, 44, 1557-1572.

Grossman, P., Niemann, L., Schmidt, S., \& Walach, H. (2004). Mindfulness-based stress reduction and health benefits: A meta-analysis. Journal of Psychosomatic Research, 57, 35-43.

Hammen, C., Rudolph, K., Weisz, R., Rao, U., \& Burge, D. (1999). The context of depression in clinic-referred youth: Neglected areas in treat- 
ment. Journal of the American Academy of Child and Adolescent Psychiatry, 38, 64-71.

Hunsley, J., \& Lee, C. M. (2007). Research-informed benchmarks for psychological treatments: Efficacy studies, effectiveness studies, and beyond. Professional Psychology: Research and Practice, 38, 21-33.

Hyman, S. E. (2001). Mood disorders in children and adolescents: An NIMH perspective. Biological Psychiatry, 49, 962-969.

Jacobson, N. S., Roberts, L. J., Berns, S. B., \& McGlinchey, J. B. (1999). Methods for defining and determining the clinical significance of treatment effects: Description, application, and alternatives. Journal of Consulting and Clinical Psychology, 67, 300-307.

Jacobson, N. S., \& Truax, P. (1991). Clinical significance: A statistical approach to defining meaningful change in psychotherapy research. Journal of Consulting and Clinical Psychology, 59, 12-19.

Kabat-Zinn, J. (1990). Full catastrophe living: The program of the Stress Reduction Clinic at the University of Massachusetts Medical Center. New York: Dell.

Kazdin, A. E. (2003). Research design in clinical psychology (4th ed.). Boston: Allyn \& Bacon.

Kolko, D. J., Brent, D. A., Baugher, M., Bridge, J., \& Birmaher, B. (2000). Cognitive and family therapies for adolescent depression: Treatment specificity, mediation, and moderation. Journal of Consulting and Clinical Psychology, 68, 603-614.

Kreft, I., \& deLeeuw, J. (1998). Introducing multilevel modeling. Thousand Oaks, CA: Sage

Miller, J. J., Fletcher, K., \& Kabat-Zinn, J. (1995). Three-year follow-up and clinical implications of a mindfulness meditation-based stress reduction intervention in the treatment of anxiety disorders. General Hospital Psychiatry, 17, 192-200.

Napoli, M., Krech, P. R., \& Holley, L. C. (2005). Mindfulness training for elementary school students: The attention academy. Journal of Applied School Psychology, 21, 99-125.

Rosenberg, M. (1989). Society and the adolescent self-image (Rev. ed.). Middletown, CT: Wesleyan University Press.
Schwartz, J. E., \& Stone, A. A. (1998). Strategies for analyzing ecological momentary assessment data. Health Psychology, 17, 6-16.

Segal, Z. V., Williams, J. M. G., \& Teasdale, J. D. (2002). Mindfulnessbased cognitive therapy for depression: A new approach to preventing relapse. New York: Guilford Press.

Semple, R. J., Reid, E. F. G., \& Miller, L. (2005). Treating anxiety with mindfulness: An open trial of mindfulness training for anxious children. Journal of Cognitive Psychotherapy, 19, 379-392.

Shaffer, D., Fisher, P., Dulcan, M., Davies, M., Piacentini, J., SchwabStone, M., et al. (1996). The NIMH Diagnostic Interview Schedule for Children Version 2.3 (DISC-2.3): Description, acceptability, prevalence rates, and performance in the MECA study. Journal of the American Academy of Child and Adolescent Psychiatry, 35, 865-877.

Shapiro, S. L., Brown, K. W., \& Biegel, G. M. (2007). Teaching self-care to caregivers: Effects of mindfulness-based stress reduction on the mental health of therapists in training. Training and Education in Professional Psychology, 1, 105-115.

Shapiro, S. L., Carlson, L. E., Astin, J. A., \& Freedman, B. (2006). Mechanisms of mindfulness. Journal of Clinical Psychology, 62, 373386

Spielberger, C. D. (1983). Manual for the State-Trait Anxiety Inventory: STAI (Form Y). Palo Alto, CA: Consulting Psychologists Press.

U.S. Department of Health and Human Services. (1999). Mental health: A report of the Surgeon General. Rockville, MD: National Institutes of Health.

U.S. Public Health Service. (2000). Report of the Surgeon General's Conference on Children's Mental Health: A national action agenda. Washington, DC: U.S. Department of Health and Human Services.

Walker, L., Greene, J., Garber, J., Horndasch, R. L., Barnard, J., \& Ghishan, F. (1993). Psychosocial factors in pediatric abdominal pain: Implications for assessment and treatment. Clinical Psychologist, 26, 206-213.

Wall, R. B. (2005). Tai chi and mindfulness-based stress reduction in a Boston public middle school. Journal of Pediatric Health Care, 19, $230-237$.

\section{Appendix}

SCL-90 Median and Range (Minimum, Maximum) Values in MBSR and TAU Groups at Pretreatment, Posttreatment, and Follow-Up Time Points for Intent-to-Treat Sample

\begin{tabular}{|c|c|c|c|c|c|c|c|c|c|c|c|c|}
\hline \multirow[b]{3}{*}{ Variable } & \multicolumn{6}{|c|}{ MBSR } & \multicolumn{6}{|c|}{ TAU } \\
\hline & \multicolumn{2}{|c|}{ Pretest } & \multicolumn{2}{|c|}{ Posttest } & \multicolumn{2}{|c|}{ Follow-up } & \multicolumn{2}{|c|}{ Pretest } & \multicolumn{2}{|c|}{ Posttest } & \multicolumn{2}{|c|}{ Follow-up } \\
\hline & $M$ & Range & $M$ & Range & $M$ & Range & $M$ & Range & $M$ & Range & $M$ & Range \\
\hline \multicolumn{13}{|l|}{ SCL-90 } \\
\hline Somatization & 15.0 & $0,38.0$ & 9.0 & $0,31.0$ & 7.0 & $0,32.0$ & 12.0 & $0,37.0$ & 12.0 & $0,39.0$ & 12.0 & $0,39.0$ \\
\hline Obsessive & 20.5 & $0,36.0$ & 12.0 & $1,32.0$ & 11.0 & $0,29.0$ & 17.0 & $0,38.0$ & 14.0 & $0,39.0$ & 14.0 & $0,38.0$ \\
\hline Interpersonal & 15.0 & $0,36.0$ & 8.0 & $0,25.0$ & 7.5 & $0,23.0$ & 15.0 & $0,32.0$ & 12.0 & $0,34.0$ & 12.0 & $0,31.0$ \\
\hline Depression & 22.5 & $0,47.0$ & 12.0 & $0,35.0$ & 10.5 & $0,38.0$ & 24.0 & $1,47.0$ & 19.0 & $0,47.0$ & 17.5 & $0,45.0$ \\
\hline Anxiety & 13.0 & $0,37.0$ & 7.0 & $0,28.0$ & 4.0 & $0,25.0$ & 10.0 & $0,35.0$ & 7.5 & $0,40.0$ & 7.5 & $0,35.0$ \\
\hline Hostility & 9.0 & $0,21.0$ & 6.0 & $0,19.0$ & 5.0 & $0,19.0$ & 9.0 & $0,24.0$ & 8.0 & $0,20.0$ & 7.0 & $0,21.0$ \\
\hline
\end{tabular}

Note. $\quad$ MBSR $=$ mindfulness-based stress reduction; TAU $=$ treatment as usual; SCL-90 $=$ Symptom Checklist-90-R

Received October 25, 2007 